\title{
POWERS OF HOMEOMORPHISMS WITH ALMOST PERIODIC PROPERTIES
}

\author{
W. H. GOTTSCHALK ${ }^{1}$
}

Let $X$ be a topological space (an "accessible space," a "1-space," or a " $T_{1}$-space" in the terminology of Fréchet, Kuratowski, or Alexandroff-Hopf, respectively) and let $f(X)=X$ be a homeomorphism. We use the following terminology, which was suggested by G. A. Hedlund and which is to be carefully distinguished from those terminologies used by Birkhoff, Ayres, Whyburn, and others. A point $x$ of $X$ is said to be recurrent under $f$ provided that to each neighborhood $U$ of $x$ there corresponds a positive integer $n$ such that $f^{n}(x) \in U$. The mapping $f$ is said to be pointwise recurrent provided that each point of $X$ is recurrent under $f$. A point $x$ of $X$ is said to be almost periodic under $f$ provided that to each neighborhood $U$ of $x$ there corresponds a monotone increasing sequence $n_{1}, n_{2}, \cdots$ of positive integers with the properties that the numbers $n_{i+1}-n_{i}(i=1,2, \cdots)$ are uniformly bounded and $f^{n i}(x) \in U(i=1,2, \cdots)$. The mapping $f$ is said to be pointwise almost periodic provided each point of $X$ is almost periodic under $f$. Following Birkhoff $\left[1\right.$, p. 198], ${ }^{2}$ a subset $Y$ of $X$ is said to be minimal under $f$ provided that $Y$ is nonvacuous, closed and invariant under $f$, that is, $f(Y)=Y$, and furthermore $Y$ does not contain a proper subset with these properties. For $x \in X$, the set $n \sum_{-\infty}^{+\infty} f^{n}(x)$ is called the orbit of $x$ under $f$ and the set $\sum_{n=0}^{+\infty} f^{n}(x)$ is called the semi-orbit of $x$ under $f$. A decomposition of $X$ is a collection of nonvacuous pairwise disjoint closed subsets of $X$ which fill up $X$.

THEOREM 1. If $x \in X$ is recurrent under $f$, then $x$ is also recurrent under $f^{n}$ for every positive integer $n$.

PRoof. We make use of an induction. The theorem is true for $n=1$. Let $m$ be any positive integer. Assume the theorem is true for $n \leqq m$. We now show the theorem is true for $n=m+1=k$.

We may suppose without loss of generality that $X$ is the closure of the semi-orbit of $x$ under $f$, for this set is invariant under $f$. Define $X_{i}(i=0,1, \cdots, k)$ to be the closure of the semi-orbit of $f^{i}(x)$ under $f^{k}$. It is readily verified that $f\left(X_{i}\right)=X_{i+1}(i=0,1, \cdots, k-1)$,

Presented to the Society, February 26, 1944; received by the editors December 8, 1943.

1 I wish to thank Professor G. A. Hedlund for his genial interest in the development of these results.

2 Numbers in brackets refer to the bibliography at the end of the paper. 
$f^{k}\left(X_{i}\right) \subset X_{i}(i=0,1, \cdots, k)$, and $X=\sum_{i=0}^{k-1} X_{i}$. We may suppose that $x \in \sum_{i=1}^{k-1} X_{i}$, for otherwise the conclusion follows. Let $p, 1 \leqq p \leqq k-1$, be the smallest integer such that $x \in X_{p}$. Then, the semi-orbit of $x$ under $f^{k}$ is contained in $X_{p}$. Hence, $X_{0} \subset X_{p}=f^{p}\left(X_{0}\right)$ and $p$ is the smallest positive integer such that $X_{0} \subset f^{p}\left(X_{0}\right)$. Since $f^{k}\left(X_{0}\right) \subset X_{0}$, there exists a smallest positive integer $t$ such that $f^{t}\left(X_{0}\right) \subset X_{0}$. Now $f^{t}\left(X_{0}\right) \subset X_{0} \subset f^{p}\left(X_{0}\right)$. Since $f^{t-p}\left(X_{0}\right) \subset X_{0}$ and $X_{0} \subset f^{p-t}\left(X_{0}\right), p$ is neither less than nor greater than $t$. Hence, $p=t$ and $f^{p}\left(X_{0}\right)=X_{0}$. Write $k=p q+r, 0 \leqq r<p$, where $q$ and $r$ are integers. Now

$$
X_{0} \supset f^{k}\left(X_{0}\right)=f^{r}\left(f^{p q}\left(X_{0}\right)\right)=f^{r}\left(X_{0}\right) .
$$

Thus, $r=0$ and $k=p q$. If $p=1$, then $X_{0}=X_{1}=\cdots=X_{k}$ whence $x \in X_{k}$ and the conclusion follows. We may suppose, therefore, that $p>1$. Now $p \leqq m$ and $q \leqq m$. By the induction assumption, $x$ is recurrent under $f^{p}$ and, applying the induction assumption to $f^{p}, x$ is recurrent under $\left(f^{p}\right)^{q}=f^{k}$.

COROLlaRY 1. Every positive power of a pointwise recurrent homeomorphism on a topological space is itself pointwise recurrent.

Theorem 1 can be used to provide a different proof of the following theorem, due to Birkhoff and Smith [2, p. 358, Theorem 3].

TheOREM. If $X$ is a compact metric space and if $f(X)=X$ is a homeomorphism, then for every nonzero integer $n$ the central orbits under $f^{n}$ are identical with the central orbits under $f$.

This follows from Theorem 1 and the result, due to Birkhoff and Smith [2, p. 353, Theorem 2], that the sum of the central orbits under a homeomorphism $h$ on a compact metric space is characterized as the closure of the set of points recurrent under both $h$ and $h^{-1}$. Although their results on central orbits [2, pp. 350-355, 356-360] are stated for closed surfaces, their proofs are actually valid for compact metric spaces.

THEOREM 2. If $X$ is a compact connected metric space and if the recurrent points are dense in $X$, then every recurrent cut point $x$ of $X$ is periodic.

Proof. Express $X=A+B$, where $A$ and $B$ are nondegenerate continua such that $A \cdot B=x$. By a theorem due to Kelley $[4$, p. 194, Theorem 3.4] there exists an $F$-set (that is, either a simple link, or cut point, or end point) $F$ of $X$ such that $f(F)=F$. (For properties of simple links, see Whyburn $[5$, pp. 64-65].) Now $F$ is contained in 
either $A$ or $B$, say $A$, and $A \cdot f^{n}(A) \neq \Lambda$ for every integer $n$. Since some point of $B-x$ is recurrent under $f$, there exists a positive integer $n$ such that $B \cdot f^{n}(B) \neq \Lambda$. By Theorem $1, x$ is recurrent under $f^{n}$. Applying a lemma due to Whyburn [5, p. 247, Lemma 4.21], it follows that $f^{n}(x)=x$. The proof is completed.

Theorem 2 and its proof are partial generalizations of Whyburn $[5$, p. 248 , Theorem 4.6$]$, but the original conclusion-that the mapping is elementwise periodic on all simple links-is no longer valid without semi-local connectedness, even though the mapping be regularly almost periodic in the sense of Whyburn $[5$, p. 250]. Theorem 1 , however, may be used as an aid in the proof of the cited theorem.

Remarks. 1. If the subset $Y$ of $X$ is minimal under $f$, then $Y$ is minimal also under $f^{-1}$. 2. A nonvacuous subset $Y$ of $X$ is minimal if and only if the closure of the orbit of every point of $Y$ is $Y$. 3. The collection of sets minimal under $f$ is a decomposition of $X$ if and only if the closure of the orbits under $f$ is a decomposition of $X$; and, in either case, these two collections coincide. In other words, $f$ gives $a$ minimal-set decomposition if and only if $f$ gives an orbit-closure decomposition.

THEOREM 3. If $X$ is minimal under $f$ but not under $f^{k}$, where $k$ is a nonzero integer, then there exists an integer $n, n>1$, such that $n$ divides $|k|$ and $f^{n}$ gives a finite minimal-set decomposition which contains exactly $n$ elements.

PROof. By Remark 1, it is sufficient to prove the theorem when $k$ is positive. There exists a point $x$ of $X$ such that the orbit of $x$ under $f^{k}$ is not dense in $X$, by Remark 2. Define $X_{i}(i=0,1, \cdots, k-1)$ to be the closure of the orbit of $f^{i}(x)$ under $f^{k}$. Clearly, $f\left(X_{i}\right)=X_{i+1}$ $(i=0,1, \cdots, k-2), f\left(X_{k-1}\right)=X_{0}$, and $f^{k}\left(X_{i}\right)=X_{i}(i=0,1, \cdots$, $k-1)$. Let $p$ be the maximum positive integer such that there exist integers $i_{1}, i_{2}, \cdots, i_{p}$ with the properties that $0 \leqq i_{1}<i_{2}<\cdots<i_{p}$ $\leqq k-1$ and $\prod_{j=1}^{p} X_{i_{j}} \neq \Lambda$. Choose integers $i_{1}, i_{2}, \cdots, i_{p}$ with these properties. Define $Y=\prod_{j=1}^{p} X_{i_{j}}$. Clearly, $f^{k}(Y)=Y$. Let $n$ be the smallest positive integer such that $f^{n}(Y)=Y$. Define $Y_{j}=f^{i}(Y)$ $(j=0,1, \cdots, n-1)$. The sets $Y_{j}(j=0,1, \cdots, n-1)$ are closed and pairwise disjoint. Choose $y \in Y$. Then,

$$
X=\overline{j \sum_{-\infty}^{+\infty} f^{i}(y)}=\overline{j \sum_{-\infty}^{+\infty} f^{i}(Y)}=\sum_{j=0}^{n-1} f^{i}(Y)=\sum_{j=0}^{n-1} Y_{j} .
$$

Thus, $D \equiv\left[Y_{j} \mid j=0,1, \cdots, n-1\right]$ is a decomposition of $X$.

We show $n>1$. Suppose $n=1$. Then, $X=Y_{0}=Y=X_{i_{1}}$, and thus 
$X=f^{k-i_{1}}\left(X_{i_{1}}\right)=f^{k}\left(X_{0}\right)=X_{0}$ whence the orbit of $x$ under $f^{k}$ is dense in $X$, contrary to the second statement of the proof.

We show that $n$ divides $k$. Write $k=q n+r, 0 \leqq r<n$, where $q$ and $r$ are integers. Then,

$$
Y=f^{k}(Y)=f^{r}\left(f^{q n}(Y)\right)=f^{r}(Y) .
$$

Hence, $r=0$.

In order to show that each element $Y_{j}$ of $D$ is minimal under $f^{n}$, it is sufficient to observe that for $y \in Y_{j}$, the orbit of $y$ under $f$ is dense in $X, D$ is a decomposition of $X$ whose elements are invariant un$\operatorname{der} f^{n}$, and the subset of the orbit of $y$ under $f$ which is contained in $Y_{j}$ is actually the orbit of $y$ under $f^{n}$.

Corollary 2. If $X$ is connected and minimal under $f$, then $X$ is also minimal under $f^{n}$ for every nonzero integer $n$.

Corollary 3. If $X$ has only finitely many, say $k$, components and if $X$ is minimal under $f$, then for every nonzero integer $n$ the mapping $f^{n}$ gives a finite minimal-set decomposition, the number of whose elements is the greatest common divisor of $k$ and $|n|$.

If $k=1$, Corollary 3 reduces to Corollary 2. If $k>1$, Corollary 3 may be proved by first of all considering the case when $k=k^{\prime}$ and $n=n^{\prime}>0$ are relatively prime and then extending the result to $k=a k^{\prime}$ and $n=a n^{\prime}$, where $a$ is any positive integer. Corollary 3 essentially combines Corollary 2 with a property of cyclic counting or, what is the same, a property of periodic orbits.

THEOREM 4. If $X$ is minimal under $f$, then for every nonzero integer $n$ the mapping $f^{n}$ gives a finite minimal-set decomposition of $X$ into at most $|n|$ elements.

Proof. By Remark 1, it is sufficient to prove the theorem when $n$ is positive. We make use of an induction. The theorem is true for $n=1$. Let $m$ be any positive integer. Assume the theorem is true for $n \leqq m$. We now show the theorem is true for $n=m+1=k$.

If $X$ is minimal under $f^{k}$, the conclusion follows. Suppose now that $X$ is not minimal under $f^{k}$. By Theorem 3 , there exist in tegers $p$ and $q$ such that $p>1, k=p q$, and $f^{p}$ gives a finite minimal-set decomposition $D$ of $X$ into exactly $p$ elements. Let $Y$ be any element of $D$. Now apply the induction assumption to $f^{p}(Y)=Y$ and $n=q \leqq m$. Thus $\left(f^{p}\right)^{q}=f^{k}$ gives a finite minimal-set decomposition of $Y$ into at most $q$ elements. Hence, $\left(f^{p}\right)^{q}=f^{k}$ gives a finite minimal-set decomposition of $X$ into at most $p q=k$ elements. 
COROLLARY 4. If the mapping $f$ gives an orbit-closure decomposition of $X$, then for every integer $n$ the mapping $f^{n}$ also gives an orbit-closure decomposition of $X$.

Proof. By virtue of Remark 3, it is sufficient to apply Theorem 4 to the elements of the orbit-closure decomposition given by $f$.

Lemma 1. If $X$ is a metric space and if $x \in X$ is almost periodic un$\operatorname{der} f$, then the closure $Y$ of the orbit of $x$ under $f$ is minimal under $f$.

Proof. Suppose $Y$ is not minimal. Then, there exists a nonvacuous closed invariant subset $Z$ of $Y$ such that $x \notin Z$. Choose $z \in Z$. Let $2 \epsilon$ be the distance from $x$ to $Z$. There exists a positive integer $N$ such that in every set of $N$ consecutive positive integers appears an integer $n$ so that $\rho\left(x, f^{n}(x)\right)<\epsilon$, where $\rho$ is the metric in $X$. Choose $\delta>0$ so small that $x^{\prime} \in X$ with $\rho\left(z, x^{\prime}\right)<\delta$ implies $\rho\left(f^{i}(z), f^{i}\left(x^{\prime}\right)\right)<\epsilon$ $(i=1,2, \cdots, N)$. There exists an integer $p \geqq 0$ such that $\rho\left(z, f^{p}(x)\right)$ $<\delta$. Also it is possible to find an integer $q, 1 \leqq q \leqq N$, so that $\rho\left(x, f^{p+q}(x)\right)<\epsilon$. Furthermore, $\rho\left(f^{q}(z), f^{p+q}(x)\right)<\epsilon$. Hence, $\rho\left(x, f^{q}(z)\right)$ $<2 \epsilon$ which is impossible because $f^{q}(z) \in Z$.

LEMMA 2. If $X$ is a compact metric space and if $f$ gives an orbitclosure decomposition, then $f$ is pointwise almost periodic.

Proof. Suppose that some point $x$ of $X$ is not almost periodic. Then there exist a neighborhood $U$ of $x$ and a sequence $m_{1}, m_{2}, \cdots$ of posi-

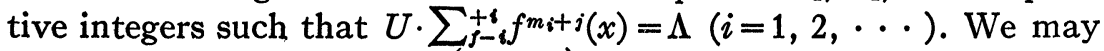
suppose that the sequence $\left\{f^{m_{i}}(x)\right\}$ converges to some point, say $y$, of $X$. It is easy to show that the orbit of $y$ is contained in $X-U$. Hence, the closure of the orbit of $y$ is a proper subset of the closure of the orbit of $x$. This is impossible.

THEOREM 5. If $X$ is a metric space, then in order that $f$ give an orbitclosure decomposition it is sufficient that $f$ be pointwise almost periodic; and in case $X$ is compact, this condition is also necessary.

The proof follows easily from Lemmas 1 and 2 and Remark 3. Theorem 5 and Lemmas 1 and 2 are closely related to Hall and Kelley $[3$, p. 628, Theorem 4] and to Birkhoff $[1$, p. 199].

THEOREM 6. Every power (including negative powers) of a pointwise almost periodic homeomorphism on a compact metric space is itself pointwise almost periodic.

The proof follows readily from Theorem 5 and Corollary 4 .

THeOREM 7. If $X$ is a compact metric space and if $x \in X$ is almost 
periodic under $f$, then $x$ is also almost periodic under $f^{n}$ for every integer $n$.

The proof proceeds easily from Lemma 1, Remark 2, and Theorems 5 and 6.

\section{BIBLIOGRAPHY}

1. G. D. Birkhoff, Dynamical systems, Amer. Math. Soc. Colloquium Publications, vol. 9, New York, 1927.

2. G. D. Birkhoff and P. A. Smith, Structure analysis of surface transformations, J. Math. Pures Appl. vol. 7 (1928) pp. 345-379.

3. D. W. Hall and J. L. Kelley, Periodic types of transformations, Duke Math. J. vol. 8 (1941) pp. 625-630.

4. J. L. Kelley, A decomposition of compact continua and related theorems on fixed sets under continuous transformations, Proc. Nat. Acad. Sci. U.S.A. vol. 26 (1940) pp. 192-194.

5. G. T. Whyburn, Analytic topology, Amer. Math. Soc. Colloquium Publications, vol. 28, New York, 1942.

UNIVERSITY OF VIRGINIA

\section{SOME PROPERTIES OF SUMMABILITY. II ${ }^{1}$}

\section{J. D. HILL}

1. Summability of bounded sequences. It follows from a well known result of $H$. Steinhaus ${ }^{2}$ that no regular matrix method of summability can be effective for (that is, assign a finite limit to) every element in the space $(m)$ of bounded sequences. The object of this note is to consider some questions suggested by this fact. The first of these may be formulated as follows. If $A$ is a given regular matrix method let $J_{A}$ denote the set of all $A$-summable bounded sequences. We then ask what are necessary and sufficient conditions on a subset $E$ of $(m)$ in order that there exist a regular $A$ such that $E \subset J_{A}$ ? In Theorem 1 below it is shown that the separability of $E$ is a sufficient condition. It seems unlikely that this condition is necessary although we have been unable to decide the question. It is clearly equivalent to the question of whether every $J_{A}$ is separable.

TheOREM 1. Let $E$ be an arbitrary separable subset of $(m)$. Then every regular matrix $A=\left(a_{m k}\right)$ contains a (necessarily regular) row-submatrix $B=\left(a_{m_{i} k}\right)$ such that $E \subset J_{B}$.

Received by the editors November 6, 1943.

1 This note is in the nature of an appendix to the paper cited in footnote 4.

$2 \mathrm{H}$. Steinhaus, Some remarks on the generalizations of the notion of limit (in Polish), Prace Matematyczno-Fizyczne vol. 22 (1921) pp. 121-134. See also I. Schur, Über lineare Transformationen in der Theorie der unendlichen Reihen, J. Reine Angew. Math. vol. 151 (1921) pp. 79-111. 